\title{
KPI-121 0.25\%: A New Option for the Treatment of Dry Eye Disease
}

\author{
Kenneth Beckman, ${ }^{1,2}$ James A Katz, ${ }^{3}$ Parag A Majmudar ${ }^{4}$ and Audrey Talley Rostov ${ }^{5}$ \\ 1. Comprehensive Eyecare of Central Ohio, Westerville, OH, USA; 2. Department of Ophthalmology, Ohio State University, Columbus, OH, USA; \\ 3. The Midwest Center for Sight, Des Plaines, IL, USA; 4. Chicago Cornea Consultants, Highland Park, IL, USA; 5. Northwest Eye Surgeons, \\ Seattle, WA, USA
}

$\mathrm{D}$ ry eye disease (DED) is a chronic inflammatory condition that, for many patients, is also characterized by acute episodic exacerbations of signs and symptoms. Until recently, treatments for DED have focused on relieving chronic symptoms by targeting the compromised lipid and aqueous layers of the tear film and the inflammation of the corneal and conjunctival epithelia. KPI-121 ophthalmic suspension $0.25 \%$ (EYSUVIS ${ }^{\circledast}$, Kala Pharmaceuticals, Inc., Watertown, MA, USA) is a new formulation of the corticosteroid loteprednol etabonate, approved for short-term use (up to 2 weeks) in the treatment of DED. This formulation uses mucus-penetrating particles to greatly enhance the delivery of loteprednol etabonate to the epithelial cells of the cornea and conjunctiva by reducing tear-film clearance of the drug. In patients with chronic DED, clinical trials have demonstrated that KPI-121 0.25\% reduces signs and symptoms of the disease following a 2-week course of treatment. The most common adverse reaction was pain at the instillation site, and the incidence of elevated intraocular pressure was low. KPI-121 0.25\% has the potential to reduce signs and symptoms of DED in patients who experience episodic exacerbations of the disease. In this review, we discuss the underlying mechanisms of DED; describe the challenge in delivering topical ocular medications to the cornea and conjunctiva; and review the efficacy, safety and mechanism of action of KPI-121 0.25\%.

\section{Keywords}

Dry eye disease, mucus-penetrating particles, nanoparticles, loteprednol etabonate, KPI-121, ocular mucus, ocular surface, exacerbations

Disclosure: Kenneth Beckman is a consultant for Alcon, Allergan, Bausch + Lomb (a division of Bausch Health US), Dompé, EyePoint Pharmaceuticals, Eyevance Pharmaceuticals, Kala Pharmaceuticals, Novartis, Ocular Science, Ocular Therapeutix and Sun Pharmaceutical Industries. James A Katz is a consultant for Alcon, Allergan, EyePoint Pharmaceuticals, Eyevance Pharmaceuticals, Kala Pharmaceuticals, Novartis, Ocular Science and Ocular Therapeutix. Parag A Majmudar is a consultant for Alcon, Allergan, Bausch + Lomb (a division of Bausch Health US), Bio-Tissue Inc., Dompé, Eyevance Pharmaceuticals, Kala Pharmaceuticals, Novaliq, Novartis and Sun Pharmaceutical Industries. Audrey Talley Rostov is a consultant for Alcon, Allergan, Bausch + Lomb (a division of Bausch Health US), Dompé, Eyevance

Pharmaceuticals, Kala Pharmaceuticals, Novartis and Sun Pharmaceutical Industries.

Acknowledgements: Editorial support was provided by Chery

McCutchan PhD; Lisa Baker, PhD; and Esther Tazartes, MS, of the Global

Outcomes Group, and was funded by Kala Pharmaceuticals, Inc.

Review process: Double-blind peer review.

Compliance with ethics: This study involves a review of the literature and did not involve any studies with human or animal subjects performed by any of the authors.

Authorship: The named authors meet the International Committee

of Medical Journal Editors (ICMJE) criteria for authorship of this

manuscript, take responsibility for the integrity of the work as a whole,

and have given final approval for the version to be published.

Access: This article is freely accessible at touchOPHTHALMOLOGY.com

(C) Touch Medical Media 2021

Received: 24 May 2021

Accepted: 26 August 2021

Published online: 26 November 2021

Citation: touchREVIEWS in Ophthalmology. 2021;15(2):58-62

Corresponding author: Kenneth Beckman, Comprehensive Eyecare of Central Ohio 450 Alkyre Run Drive \#100, Westerville, OH 43082, USA. E: kenbeckman22@aol.com

Support: No funding was received for the publication of this article.
Dry eye disease (DED) is an inflammatory condition of the cornea and conjunctiva that is characterized by tear-film instability and increased tear osmolarity. ${ }^{1-3}$ While DED is traditionally viewed as a chronic condition, many patients experience episodic exacerbations of the disease, also referred to as flares or episodic dry eye.' Until recently, the need for an effective treatment for DED with rapid onset has remained unmet. In October 2020, KPI-121 ophthalmic suspension 0.25\% (EYSUVIS ${ }^{\oplus}$, Kala Pharmaceuticals, Inc., Watertown, MA, USA) was approved in the US for the short-term (up to 2 weeks) treatment of the signs and symptoms of DED. ${ }^{4,5}$ This ophthalmic formulation of loteprednol etabonate uses mucus-penetrating particles (MPPs) that are able to deliver greater concentrations of corticosteroid to the anterior ocular tissues compared with conventional ophthalmic suspensions. ${ }^{6}$ In this review, we discuss the underlying mechanisms of DED, focusing specifically on the inflammatory nature of the disease and periodic exacerbations of signs and symptoms. We also describe the challenge in delivering topical ocular medications to the corneal and conjunctival epithelia. Finally, we review the efficacy, safety and mechanism of action of KPI-121 0.25\%.

\section{Dry eye disease}

DED is one of the most common ocular disorders, with diagnosed DED affecting $6.8 \%$ of the US adult population, or $\sim 16.4$ million people.? Intrinsic and extrinsic factors influence the prevalence of DED, which increases from $3.4 \%$ in adults under the age of 40 to $11.3 \%$ in those over the age of 50 , and is more common in persons of Asian descent. ${ }^{2.7}$ The prevalence of DED is similar in men and women between the ages of 18 and 34, but increases more rapidly with age in women than in men. ${ }^{7-9}$ Extrinsic factors that have been shown to exacerbate DED include environmental conditions (e.g. low humidity, wind, heaters or air conditioning) and specific activities (e.g. spending time at a computer, reading or watching television). ${ }^{10-15}$

The tear film consists of the lipid layer, the aqueous layer and the mucous layer, which is in direct contact with the corneal and conjunctival epithelia (Figure 1). ${ }^{16}$ Both the mucous layer and the aqueous layer contain mucins, which are high-molecular-weight glycoproteins prone 
Figure 1: Structure of the tear film and corneal epithelium of the eye

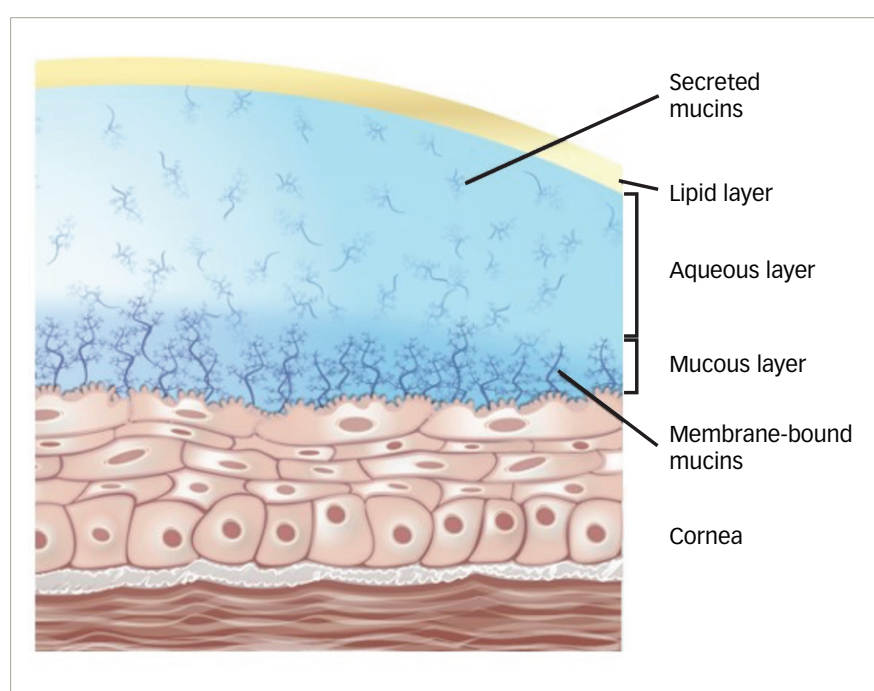

The tear film consists of a lipid layer, which is secreted by the meibomian glands; the aqueous layer, which is secreted by the lacrimal gland; and the mucous layer, which is secreted by the cells of the epithelium. The mucous layer is composed of membranebound mucins. In addition to the membrane-bound mucins, the epithelium secretes mucins found in the aqueous layer, which are referred to as secreted mucins. Reused with permission from Gupta P, Venkateswaran N. 2021. ${ }^{16}$

to strong adhesive interactions and the formation of fibrous, gel-like structures. The mucous layer is composed of membrane-associated mucins, which form a dense glycocalyx matrix on the apical surfaces of the cornea and conjunctiva, and primarily serve to protect the underlying tissues from abrasive stress. Additionally, the membranebound mucins protect the ocular surface epithelia from pathogens and extraneous molecules by providing a barrier to penetration. The mucins in the aqueous layer, which are referred to as secreted mucins, are produced predominantly by conjunctival goblet cells and released into the tear film. These secreted mucins play a major role in trapping foreign objects, such as allergens, pathogens and debris, via mucoadhesive interactions. ${ }^{6}$ Once bound, these substances move with the tear film ,and are rapidly cleared from the ocular surface via tear-fluid turnover, nasolacrimal drainage and blinking. ${ }^{17}$

DED is characterized by an unstable tear film that is associated with inflammation, damage to the ocular surface and neurosensory abnormalities. ${ }^{2,3}$ Key aetiological features include tear-film instability, tear hyperosmolarity and inflammation of the ocular surface. Historically, DED has been categorized as either aqueous deficient, defined as decreased tear production by the lacrimal gland, or as evaporative, characterized by a reduction of the tear film through evaporation. ${ }^{3}$ Treatment for DED has been based on increasing tear production, reducing evaporation and lid hygiene. In mild cases of DED, these treatment approaches may be sufficient to reduce the signs and symptoms of DED.

Regardless of whether DED is characterized as aqueous deficient or evaporative, inflammation plays a key role in its pathogenesis. Inflammation of the cornea and conjunctiva begins in response to tear hyperosmolarity. ${ }^{1}$ Pro-inflammatory compounds, such as matrix metalloproteinases (MMPs) and cytokines, are secreted, and immune cells present in the tear film initiate an innate immune response, which then begins the self-perpetuating inflammatory cycle characteristic of chronic DED. ${ }^{1,18}$ In this cycle, a prolonged innate immune response can trigger an adaptive immune response. ${ }^{1}$ Thus, the ocular immune system of patients with chronic DED is primed to respond quickly to new DED triggers. The presence of a self-promoting inflammatory cycle suggests that treating ocular surface inflammation may help patients exit this cycle. 18,19

\section{Diagnosis of dry eye disease}

A clinical diagnosis of DED is made by assessing quantitative and qualitative characteristics of the tear film, ocular surface and patientreported symptoms. Diagnostic signs of DED include tear-film breakup time (TBUT; normally $>10$ seconds in a healthy eye), the pattern of tearfilm breakup, tear volume and levels of pro-inflammatory biomarkers. ${ }^{2,20,21}$ Tear osmolarity is one of the best predictors of DED severity, and variability in osmolarity is often greater in patients with DED. ${ }^{20}$ Ocular staining techniques, which are widely used to diagnose DED, are able to detect mucus thinning and damaged or dead epithelial cells. ${ }^{20}$ Patients with DED also present with symptoms of visual disturbance and ocular discomfort and/or pain, which are self-reported during office visits. ${ }^{3}$

Patients may present with signs exclusive of symptoms, symptoms exclusive of signs, or a combination of both signs and symptoms, which can complicate diagnosis of DED. In a classic study of patients with DED, no correlation was found between signs and symptoms of the disease, and clinical tests were unable to predict the occurrence or severity of symptoms. ${ }^{22}$ Furthermore, a systematic review of 33 studies that measured signs and symptoms of DED found low or inconsistent correlations between clinical measures and reported symptoms. ${ }^{23}$ These studies emphasize the potential complexity of DED diagnosis for the clinician.

\section{Episodic exacerbations in dry eye disease}

Although DED is often a chronic condition, many patients experience episodic exacerbations (flares) of the disease. ${ }^{2}$ DED exacerbations are rapidonset, inflammation-driven increases of DED signs and symptoms that are not managed adequately with long-term maintenance therapies..$^{24}$ Acute exacerbations in DED are much like those observed in other inflammatory disorders, including asthma, systemic lupus erythematosus and rheumatoid arthritis. The treatment of asthma exacerbations often includes the use of corticosteroids, ${ }^{25}$ and exacerbations of systemic lupus erythematosus may be treated with immunosuppressive agents or corticosteroids. ${ }^{26}$ DED has characteristics similar to other chronic inflammatory conditions, including periodic clinical exacerbations, that would benefit from short-term therapies like those used in asthma and lupus.

A growing body of evidence links specific activities and environmental conditions with exacerbations of DED signs and symptoms. In one study, patients with DED over the age of 50 years were assessed at baseline and after 30 minutes of reading. ${ }^{12}$ Ocular Surface Disease Index scores, epithelial staining and TBUT worsened after reading, and the intensity of the change in epithelial staining and TBUT correlated with the values of these measures at baseline. ${ }^{12}$ In two studies, patients with DED reported their symptoms and recorded environmental conditions or activity type. 11,15 Low humidity, windy conditions, air conditioning and television viewing exacerbated DED symptoms. ${ }^{11,15}$ Surface-level ozone concentrations also correlate with worsening DED symptoms. ${ }^{27}$

In studies using a controlled environment, participants also experienced an increase in the signs associated with DED. Standardized dry, breezy conditions resulted in a decrease in TBUT and an increase in corneal staining and proinflammatory biomarkers, but little or no change in symptoms. ${ }^{10,13,14}$ Regardless of disease severity, episodic exacerbations are common among patients with DED, and require targeted management. ${ }^{2}$ In mild DED cases, ongoing symptoms can often be controlled with more conservative methods, including environmental modifications and the use of artificial tears, but intermittent exacerbations may still require 
Figure 2: Comparison of conventional $(A)$ and mucus-penetrating $(B)$ topical drug delivery to the ocular surface

A
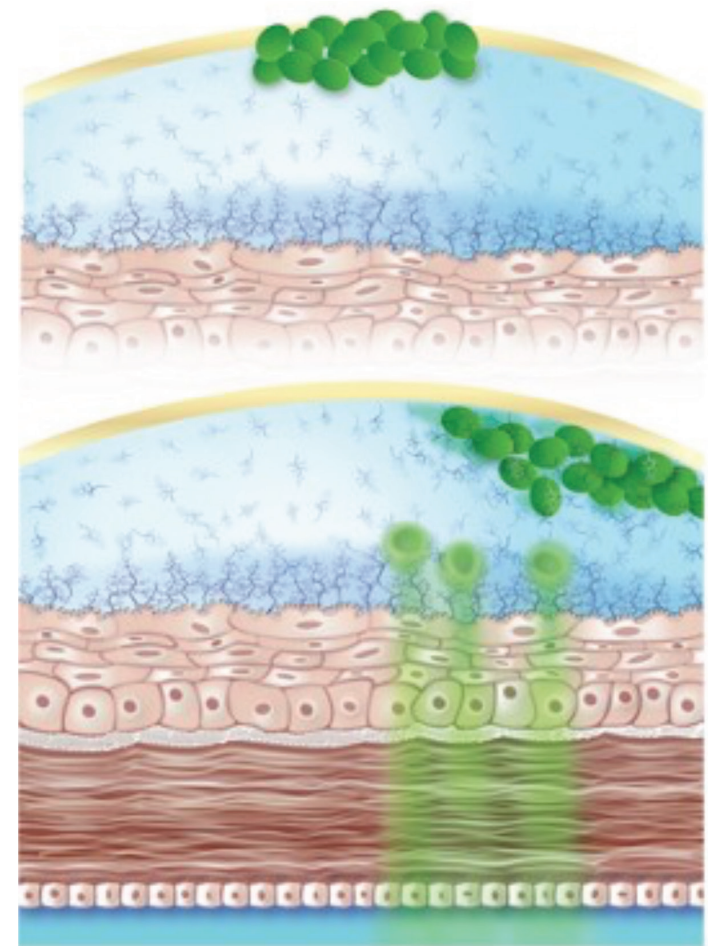

B
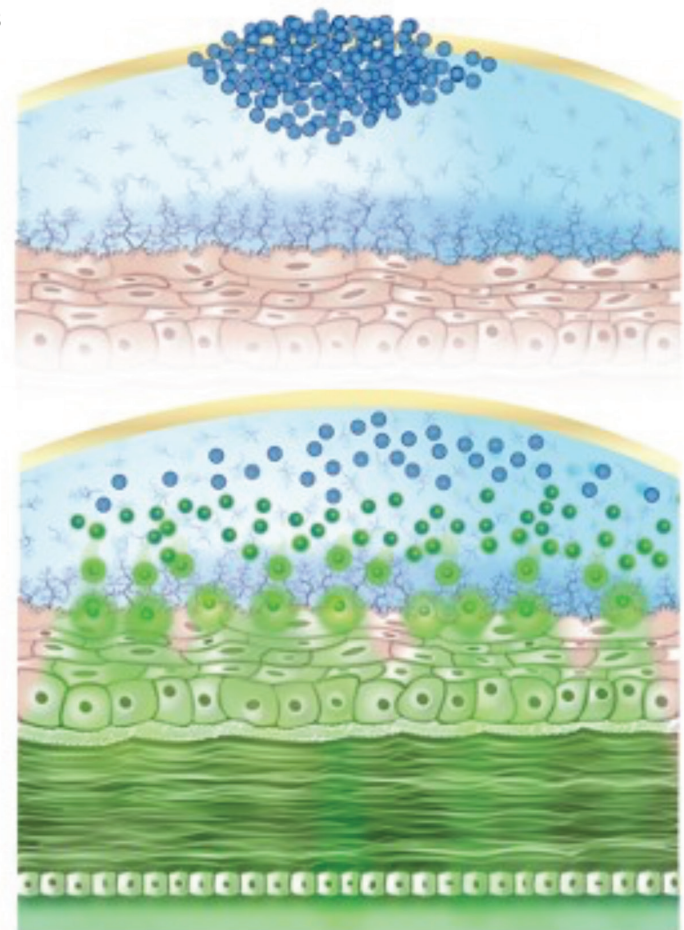

A. Conventional delivery results in the rapid clearing of topical medications via movement of the tear film across the ocular surface. A small fraction of the drug reaches and penetrates the cornea. B. Mucus-penetrating particle delivery allows topical medication to reach and move through the mucous layer, increasing the amount of medication that reaches and is absorbed by the cornea. Reused with permission from Gupta P, venkateswaran N. 2021.16

effective short-term treatment. Symptoms in patients with moderate-tosevere DED are typically controlled with chronic medication, but these patients may, nonetheless, experience acute exacerbations that require additional treatment. Thus, there is a need for effective treatment of clinical exacerbations brought on by DED triggers. ${ }^{2}$

\section{Topical ocular drug delivery and the mucus barrier}

While the tear film and mucus barrier provide excellent protection from extraneous substances that may damage the eye, this defensive mechanism also makes it challenging to deliver topical medications to the cornea and conjunctiva (Figure 2A). ${ }^{16}$ The tear film is able to clear traditional eye drops within minutes, which provides little time for a drug to penetrate the mucus barrier and reach the epithelial tissues. ${ }^{28}$ Less than $5 \%$ of a therapeutic agent administered topically reaches the anterior eye tissues. $., 17,28$

To penetrate the mucus barrier effectively, drug molecules in solution formulations or drug particles in suspension formulations should not bind to secreted or membrane-bound mucins or be too large to pass through the mucus barrier (Figure 2B). 16,29 Various eye-drop formulations have attempted to slow drug clearing using mucoadhesive microand nanoparticles, polymer-based solutions and viscous gels. ${ }^{6}$ While mucoadhesive formulations slow clearance and increase residence time of the therapeutic agent, they do not overcome entrapment by secreted or membrane-associated mucins. ${ }^{6,17}$ Furthermore, microparticles are too large $(>500 \mathrm{~nm})$ to effectively penetrate the mucin layer covering the anterior tissues of the eye, and even nanoparticles small enough to pass through the pores of the mucus barrier may interact with mucins adhesively and not reach the epithelium. ${ }^{\circ}$

\section{Mucus-penetrating particles}

MPPs have been designed specifically to overcome delivery challenges associated with the mucus barrier. The first-generation MPPs were polymeric nanoparticles ranging in size from 100 to $500 \mathrm{~nm}$ and were coated with a dense layer of low-molecular-weight polyethylene glycol. ${ }^{29-32}$ These nanoparticles presented a nearly neutral surface charge and had diffusion rates in ex vivo mucus up to three orders of magnitude greater than those of uncoated particles. ${ }^{29}$ In addition, only a small percentage of the coated particles became completely immobilized in the ex vivo mucus matrix: $<0.5 \%$ versus $32-45 \%$ for uncoated particles. ${ }^{29}$ Preclinical studies have shown that the enhanced ability of MPPs to penetrate mucus results in significantly improved distribution and prolonged retention in mucosal surfaces in various organs, including the gastrointestinal, respiratory and cervicovaginal tracts. ${ }^{33-36}$

Recently, drug-core MPPS (AMPPLIFY® Drug Delivery Technology, Kala Pharmaceuticals, Inc.), which consist largely of a therapeutic agent coated with a mucus-penetrating surface layer, have been developed (Figure 3). ${ }^{6}$ In addition to their ability to rapidly penetrate mucus, drugcore MPPs can be formulated as shelf-stable, ready-to-use aqueous suspensions using inactive ingredients generally recognized as safe or, specifically for ophthalmic applications, ingredients with a history of clinical use in eye drops. In preclinical studies, drug-core MPP technology greatly increased the penetration and availability of drugs to the ocular surface of rabbits and mini pigs. .7,38 $^{3}$

Preclinical studies, conducted by Schopf and colleagues, demonstrate the advantage of delivering loteprednol etabonate via MPPs to the ocular surface. ${ }^{37,38} \mathrm{~A}$ single ocular administration of either loteprednol etabonate conventional ophthalmic suspension $0.5 \%$ or loteprednol etabonate 


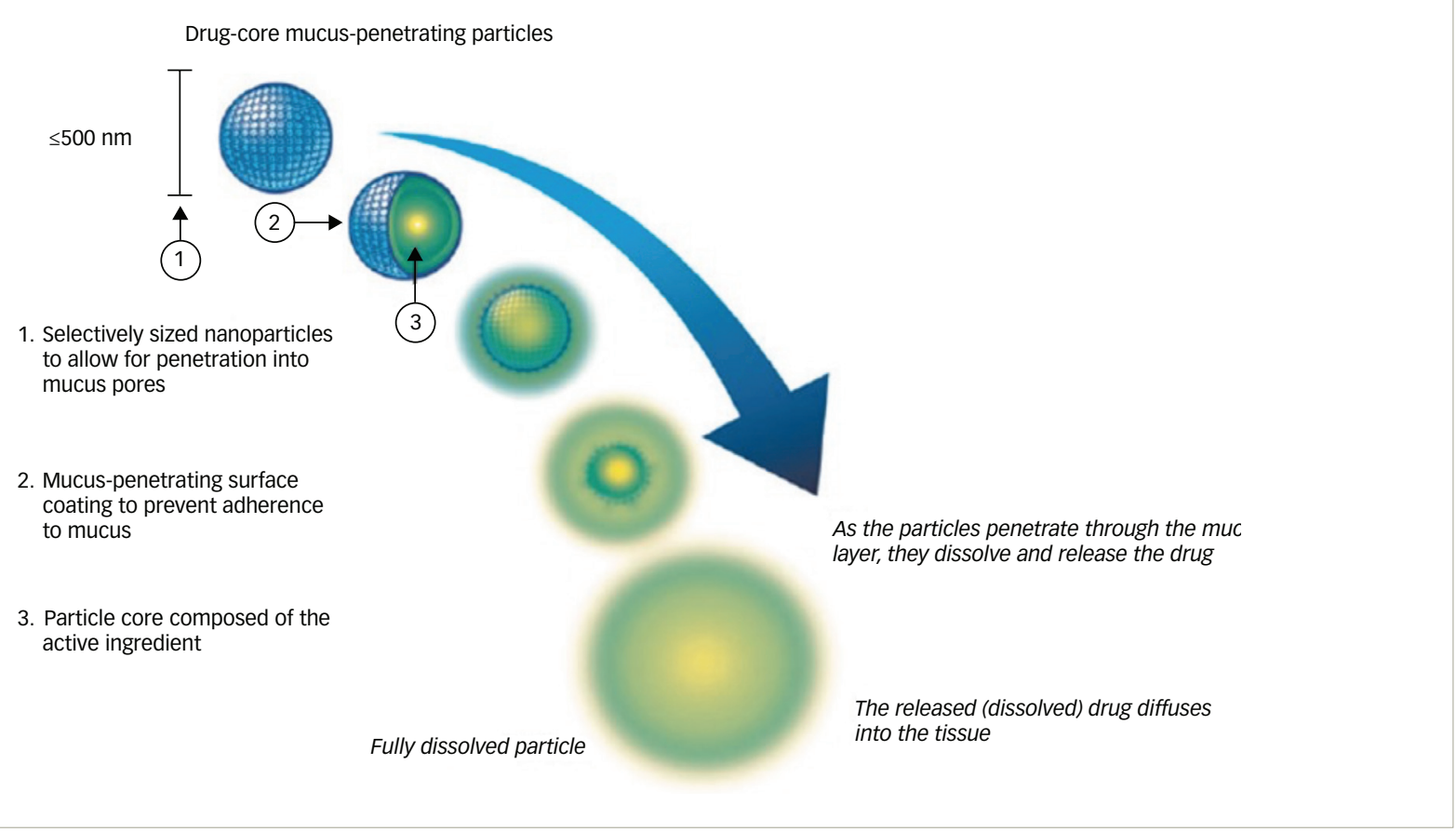

Mucus-penetrating particles are engineered to move through the aqueous and mucous layers, avoiding entrapment by secreted and membrane-bound mucins. Reused with permission from Popov $2020 .{ }^{6}$

ophthalmic suspension $0.4 \%$ formulated with MPP technology was given to rabbits. The peak concentrations of loteprednol etabonate produced by the suspension with MPP technology were 3.6 times higher in the cornea and 2.6 times higher in the conjunctiva compared with those produced by the conventional suspension. ${ }^{37}$ In another study, drug levels in the cornea of rabbits following a single instillation of $0.5 \%$ loteprednol etabonate formulated as MPPS or similarly sized nanoparticles without the MPP surface layer were compared..$^{36}$ The MPP formulation resulted in a peak corneal concentration approximately three-fold higher than that produced by the nanoparticles without the MPP surface layer. In addition, the area under the concentration-time curve was four-fold higher in the cornea in the case of the MPP formulation. ${ }^{38}$

\section{KPI-121 0.25\%: Development and clinical trials}

$\mathrm{KPI}-1210.25 \%$ is a topical ophthalmic suspension formulation developed by Kala Pharmaceuticals, Inc. that uses MPP technology to efficiently deliver the corticosteroid loteprednol etabonate to the ocular surface. Loteprednol etabonate in KPI-121 $0.25 \%$ is a retrometabolically engineered steroid that, in human tissues, breaks down rapidly into inactive metabolites, and thus may reduce the adverse events often associated with ocular corticosteroid use, which include an increased risk of developing high intraocular pressure (IOP), cataracts and glaucoma. ${ }^{39-41}$ In addition, loteprednol etabonate in KPI-121 0.25\% exhibits excellent affinity for corticosteroid-glucocorticoid receptors, has a high therapeutic index, and is lipophilic, which allows loteprednol etabonate to interact with and reduce the inflammatory response of epithelial cells in the cornea and conjunctiva. ${ }^{42}$ In a recent study, a 1-month course of treatment with loteprednol etabonate in $\mathrm{KPI}-1210.25 \%$ in combination with $0.15 \%$ sodium hyaluronate reduced signs and symptoms of DED. ${ }^{21}$ Moreover, patients with elevated MMP-9, a pro-inflammatory compound associated with epithelial damage and an increased immune response, exhibited greater reduction in signs and symptoms of DED than those with normal MMP-9 levels. ${ }^{21}$
In October 2020, the US Food and Drug Administration (FDA) approved KPI-121 0.25\% (EYSUVIS) for the short-term (up to 2 weeks) treatment of signs and symptoms of DED. ${ }^{4,5}$ The short-term relief in dry eye (STRIDE) 1 (NCT02813265), STRIDE 2 (NCT02819284) and STRIDE 3 (NCT03616899) phase III clinical trials measured the effect of KPI-121 $0.25 \%$ over a 2-week course of treatment in approximately 2,700 patients with a clinical diagnosis of DED. Collectively, these vehicle-controlled, doubleblind, multicentre trials measured ocular discomfort and change from baseline in bulbar conjunctival hyperaemia as primary endpoints. Conjunctival hyperaemia was significantly reduced compared with vehicle at the end of the 2-week trial period in all three STRIDE trials, whereas ocular discomfort was significantly reduced in the STRIDE 1 and STRIDE 3 trials, with a trend toward improvement in the STRIDE 2 trial. ${ }^{43}$ In the STRIDE trials and an additional phase II trial (NCT02188160), the most common adverse reaction was pain at the instillation site, reported by $5.2 \%$ of patients in the KPI- $1210.25 \%$ group compared with $4.4 \%$ of patients in the vehicle group. ${ }^{44}$ While elevated IOP is associated with use of ophthalmic corticosteroids, the incidence of elevated IOP was low (0.6\%) in patients receiving $\mathrm{KPI}-1210.25 \%$ compared with those receiving vehicle only (0.3\%). ${ }^{44}$ Across the studies, no severe adverse events were considered to be related to $\mathrm{KPI}-1210.25 \%$ treatment, although $10(0.7 \%)$ and $5(0.3 \%)$ patients receiving KPI-121 0.25\% and vehicle, respectively, withdrew from the studies. ${ }^{44}$ Treatment-related adverse events that led to withdrawal consisted of one incident each of conjunctival hyperaemia, conjunctival oedema, eye pruritus, ocular hyperaemia, headache, drug hypersensitivity and dyspnoea. ${ }^{44}$

\section{$\mathrm{KPI}-1210.25 \%$ in the treatment of dry eye disease}

$\mathrm{KPI}-1210.25 \%$ is indicated for the short-term (up to 2 weeks) treatment of the signs and symptoms of DED. This topical therapy is applied at a dosage of 1 to 2 drops per eye, four times per day for up to 2 weeks. ${ }^{5}$ 
Results from the STRIDE trials suggest that KPI-121 0.25\% may help reduce signs and symptoms of DED in patients with mild to severe disease who experience episodic exacerbations. In patients with mild DED who do not require long-term treatment, $\mathrm{KPI}-1210.25 \%$ may reduce exacerbations of DED that occur on a seasonal or episodic basis. Moreover, in patients with moderate-to-severe DED who are receiving long-term therapy, $\mathrm{KPI}-1210.25 \%$ would likely reduce the signs and symptoms of periodic breakthrough exacerbations.

\section{Conclusion}

Patients with DED commonly experience episodic exacerbations in symptoms that may improve with short-term therapy. KPI-121 0.25\% ophthalmic suspension, which is formulated using MPP drug-delivery technology, is approved for short-term use in DED. This new, topical ophthalmic agent significantly and rapidly reduces signs and symptoms of DED with minimal adverse effects. $\square$
. Perez VL Stern ME, Pflugfelder SC. Inflammatory basis for $\mathrm{dry}$ eye disease flares. Exp Eye Res. 2020;201:108294.

. Tsubota K Pflugfelder SC Liu Z et al. Defining dry eye from clinical perspective. Int J Mol Sci. 2020;21:9271.

3. Craig JP, Nichols KK, Akpek EK, et al. TFOS DEWS II definition and classification report. Ocul Surf. 2017;15:276-83.

4. Kala Pharmaceuticals. Kala Pharmaceuticals announces FDA acceptance of New Drug Application for EYSUVISTM for dry eye disease. 2020. Available at: https://investors.kalarx.com/newsreleases/news-release-details/kala-pharmaceuticals-announcesfda-acceptance-new-drug (accessed 27 September 2021).

5. Kala Pharmaceuticals. EYSUVIS [package insert]. Available at: www.accessdata.fda.gov/drugsatfda_docs/
label/2020/210933s000lbl.pdf (accessed 19 October 2021)

6. Popor A. Mucus-penetrating particles and the role of $0 \mathrm{cular}$ mucus as a barrier to micro- and nanosuspensions. mucus as a barrier to micro- and nanosuspe
J Ocul Pharmacol Ther 2020;36:366-75.

7. Farrand KF, Fridman M, Stillman IO, Schaumberg DA. Prevalence of diagnosed dry eye disease in the United States among adults aged 18 years and older. Am J Ophthalmol. 2017;182:90-8.

8. Schaumberg DA, Dana R, Buring JE, Sullivan DA. Prevalence of dry eye disease among US men: estimates from the Physicians' Health Studies. Arch Ophthalmol. 2009;127:763-8.

9. Schaumberg DA, Sullivan DA, Buring JE, Dana MR. Prevalence of dry eye syndrome among US women. Am I Ophthalmol. 2003; 136:318-26.

10. Fernández I, López-Miguel A, Enríquez-de-Salamanca A, et al. Response profiles to a controlled adverse desiccating environment based on clinical and tear molecule changes. OCu Surf. 2019;17:502-15

11. Amparo F, Dana R. Web-based longitudinal remote assessment of dry eye symptoms. Ocul Surf. 2018:16:249-53.

12. Karakus $S$, Agrawal $D$, Hindman $H B$, et al. Effects of prolonged reading on dry eye. Ophthalmology. 2018:125:1500-5.

13. López-Miguel A, Tesón $M$, Martín-Montañez $V$, et al. Dry eye exacerbation in patients exposed to desiccating stress unde controlled environmental conditions. Am J Ophthalmol 2014;157:788-98.

14. Tesón M, González-García MJ, López-Miguel A, et al. Influence of a controlled environment simulating an in-flight airplane cabin on dry eye disease. Invest Ophthalmol Vis SC. 2013;54:2093-9.

15. Iyer JV, Lee SY, Tong L. The dry eye disease activity log study ScientificWorldJournal. 2012;2012:589875.

16. Gupta PK, Venkateswaran N. The role of KPI-121 $0.25 \%$ in the treatment of dry eye disease: penetrating the mucus barrier to treat periodic flares. Ther Adv Ophthalmol. 2021:13.25158414211012797.

17. Taherali F, Varum F, Basit AW. A slippery slope: on the origin, role and physiology of mucus. Adv Drug Deliv Rev. 2018;124:16-33.

18. Pflugfelder SC, de Paiva CS. The pathophysiology of dry eye disease: what we know and future directions for research. Ophthalmology. 2017;124(Suppl. 11):S4-13.

19. Rolando M, Zierhut M, Barabino S. Should we reconsider the classification of patients with dry eye disease? Ocul Immunol Inflamm. 2021;29:521-3.

20. Wolffsohn JS, Arita R, Chalmers R, et al. TFOS DEWS II Diagnostic Methodology report. Ocul Surf. 2017;15:539-74.

21. Ryu KJ, Kim S, Kim MK, et al. Short-term therapeutic effects of topical corticosteroids on refractory dry eye disease: clinical prediction marker. Clin Ophthalmol. 2021:15:759-67.

22 Nichols KK Nichols IJ Mitchell GL. The lack of association between signs and symptoms in patients with dry eye disease Cornea. 2004:23:762-70

23. Bartlett JD, Keith MS, Sudharshan L, Snedecor SJ. Associations between signs and symptoms of dry eye disease: a systematic review. Clin Ophthalmol. 2015;9:1719-30.

24. ASCRS Ophthalmic Corporation. Therapeutic solutions: ocular surface disease and episodic flares of dry eye disease. Eye World. 2019:2-3. Available at: https://supplements.eyeworld. org/i/1109752-ew-may-2019-supported-by-kala-medicalaffairs/1? (accessed 19 October 2021).

25. Castillo JR, Peters SP, Busse WW. Asthma exacerbations: pathogenesis, prevention and treatment. I Allergy Clin Immunol Pract 2017:5:918-27.

26. Fanouriakis A, Kostopoulou M, Alunno A, et al. 2019 update of the EULAR recommendations for the management of systemic lupus erythematosus. Ann Rheum Dis. 2019;78:736-45.

27. Kim Y, Paik HJ, Kim MK, et al. Short-term effects of ground-leve ozone in patients with dry eye disease: a prospective clinical study. Cornea. 2019;38:1483-8.

28. Ruponen M, Urtti A. Undefined role of mucus as a barrier in ocular drug delivery. Eur $J$ Pharm Biopharm. 2015;96:442-6.

29. Lai SK, Wang YY, Hanes J. Mucus-penetrating nanoparticles for drug and gene delivery to mucosal tissues. Adv Drug Deliv Rev. 2009;61:158-71

30. Wang YY, Lai SK, Suk JS, et al. Addressing the PEG mucoadhesivity paradox to engineer nanoparticles that "slip" through the human mucus barrier. Angew Chem Int Ed Eng. 2008:47:9726-9.

31. Yang M, Lai SK, Wang YY, et al. Biodegradable nanoparticles composed entirely of safe materials that rapidly penetrate human mucus Angew Chem Int Ed Engl. 2011:50:2597-600.

32. Tang BC, Dawson M, Lai SK, et al. Biodegradable polymer nanoparticles that rapidly penetrate the human mucus barrier. Proc Natl Acad Sci U S A. 2009:106:19268-73

33. Maisel K, Ensign L, Reddy M, et al. Effect of surface chemistry on nanoparticle interaction with gastrointestinal mucus and distribution in the gastrointestinal tract following oral and rectal administration in the mouse. I Control Release. 2015;197:48-57.

34. Suk JS, Kim AJ, Trehan K, et al. Lung gene therapy with highly compacted DNA nanoparticles that overcome the mucus barrier. I Control Release. 2014;178:8-17.

35. Popov A, Schopf L, Bourassa J, Chen H. Enhanced pulmonary delivery of fluticasone propionate in rodents by mucuspenetrating nanoparticles. Int J Pharm. 2016:502:188-97.

36. Ensign LM, Tang BC, Wang YY, et al. Mucus-penetrating nanoparticles for vaginal drug delivery protect against herpes simplex virus Sci Transi Med. 2012:4:138ra79.

37. Schopf $L$, Enlow E, Popov A, et al. Ocular pharmacokinetics of a novel loteprednol etabonate $0.4 \%$ ophthalmic formulation. Ophthalmol Ther. 2014;3:63-72.

38. Schopf LR, Popov AM, Enlow EM, et al. Topical ocular drug delivery to the back of the eye by mucus-penetrating particles. Trans/ Vis Sci Technol. 2015;4:11.

39. Alberth M, Wu WM, Winwood D, Bodor N. Lipophilicity, solubility and permeability of loteprednol etabonate: a novel, soft antiinflammatory corticosteroid. J Biopharma Sci. 1991:2:115-25.

40. Bodor N, Loftsson T, Wu WM. Metabolism, distribution, and transdermal permeation of a soft corticosteroid, lotepredno etabonate. Pharm Res. 1992:9:1275-8.

41. MCGhee CN, Dean S, Danesh-Meyer H. Locally administered ocular corticosteroids: benefits and risks. Drug Saf. 2002;25:33-55.

42 Comstock TL Sheppard JD. Loteprednol etabonate for inflammatory conditions of the anterior segment of the eye: twenty years of clinical experience with a retrometabolically designed corticosteroid. Expert Opin Pharmacother. 2018;19:337-53

43. Holland E, Nichols K, Foulks G, et al. Efficacy and safety of KP$1210.25 \%$ for short term relief in dry eye (STRIDE). presented at: ASCRS-ASOA Symposium \& Congress. 16-17 May 2020.

44. Korenfeld M, Nichols KK, Goldberg D, et al. Safety of KPI-121 ophthalmic suspension $0.25 \%$ in patients with dry eye disease: a pooled analysis of 4 multicenter, randomized, vehiclecontrolled studies. Cornea. 2020;40:164-70. 